\title{
Inferences about action engage action systems
}

\author{
Lawrence J. Taylor ${ }^{\mathrm{a}, *}$, Shiri Lev-Ari ${ }^{\mathrm{b}}$, Rolf A. Zwaan ${ }^{\mathrm{a}}$ \\ a Department of Psychology, Erasmus Universiteit Rotterdam, T-Building, Room T13-20, Burg. Oudlaan 50, NL-3000 DR Rotterdam, Netherlands \\ ${ }^{\mathrm{b}}$ The University of Chicago, Department of Psychology, 5848 South University Avenue, Chicago, IL 60637, USA
}

Accepted 13 August 2007

Available online 1 November 2007

\begin{abstract}
Verbal descriptions of actions activate compatible motor responses [Glenberg, A. M., \& Kaschak, M. P. (2002). Grounding language in action. Psychonomic Bulletin \& Review, 9, 558-565]. Previous studies have found that the motor processes for manual rotation are engaged in a direction-specific manner when a verb disambiguates the direction of rotation [e.g. "unscrewed;" Zwaan, R. A., \& Taylor, L. (2006). Seeing, acting, understanding: Motor resonance in language comprehension. Journal of Experimental Psychology: General, 135, 1-11]. The present experiment contributes to this body of work by showing that verbs that leave direction ambiguous (e.g. "turned") do not necessarily yield such effects. Rather, motor resonance is associated with a word that disambiguates some element of an action, as meaning is being integrated across sentences. The findings are discussed within the context of discourse processes, inference generation, motor activation, and mental simulation.
\end{abstract}

(C) 2007 Elsevier Inc. All rights reserved.

Keywords: Discourse processes; Inference generation; Motor resonance; Mental simulation; Embodied cognition

\section{Introduction}

An emerging view in cognitive science holds that action understanding relies on the systems that are responsible for the performance of actions (Keysers \& Perrett, 2004; Rizzolatti, Fogassi, \& Gallese, 2001). In primates, the mirror neuron system (MNS), which includes neurons in the primary motor cortex, pre-motor cortex, and inferior parietal lobule (Rizzolatti \& Craighero, 2004), is activated when an individual grasps an object, observes a conspecific grasping an object (di Pellegrino, Fadiga, Fogassi, Gallese, \& Rizzolatti, 1992; Gallese, Fadiga, Fogassi, \& Rizzolatti, 1996), or sees a hand go behind a screen that occludes an object (Umiltà et al., 2001). In humans, such activation has been shown to occur when a given action falls within that individual's action repertoire (Buccino et al., 2004; Calvo-Merino, Glaser, Grezes, Passingham, \& Haggard, 2005). Similarly, the retrieval of episodic memories can

\footnotetext{
* Corresponding author. Fax: +31 104089009.

E-mail address: taylorjn@fsw.eur.nl (L.J. Taylor).
}

be facilitated if an individual replicates the posture that she had during encoding (Dijkstra, Kaschak, \& Zwaan, 2007). This research lends credence to the notion that the representations of actions rely on the MNS.

These results converge with theories proposing a strong link between the neural systems for action and the conceptual understanding of actions (Fischer \& Zwaan, in press; Gallese, 2003; Gallese \& Lakoff, 2005; Prinz, 1997; Rizzolatti \& Craighero, 2004; Wilson \& Knoblich, 2005). This leads to the prediction that the MNS is recruited to understand language that describes actions (Rizzolatti \& Arbib, 1998). Indeed, previous studies have shown that sentences describing simple motor actions both facilitate compatible motor responses (Glenberg \& Kaschak, 2002) and activate the brain regions that are active when similar actions (i.e. those that involve the same effector) are performed (de Vega, Robertson, Glenberg, Kaschak, \& Rinck, 2004; Tettamanti et al., 2005) or observed (Aziz-Zadeh, Wison, Rozzolatti, \& Iacoboni, 2006). Other results have shown that individual words can be associated with subtle, but immediate behavioral (Zwaan \& Taylor, 2006) and neural effects (Hauk, Johnsrude, \& Pulvermüller, 2004; Pulver- 
müller, Hauk, Nikulin, \& Ilmoniemi, 2005). For example, the presentation of an irrelevant word (e.g. "large" or "small") subtly influences the dynamics of a goal-directed action, such as the grip aperture of a participant's hand, as she is reaching for an object with the intention of grasping it (Gentilucci \& Gangitano, 1998; Glover \& Dixon, 2002). Analogous effects have been demonstrated for incidentally presented nouns, such as "baseball" or "tweezers," that are either larger or smaller than the target object (Glover, Rosenbaum, Graham, \& Dixon, 2004). These effects offer support to the claim that words and the affordances (Gibson, 1979) of the objects to which they can refer produce subtle, but immediate effects in the motor system (or MNS) of a comprehender.

If motor activation plays a role in language comprehension, it should occur not only after the presentation of individual words or entire sentences, but also during the comprehension of sentences. Interestingly, some experiments have demonstrated a high degree of temporal resolution with regards to action sentences inducing motor resonance in participants. Motor responses that are compatible with a described action have been found to be facilitated as soon as constraining information becomes available, before an entire sentence has been read (Chambers, Tanenhaus, Eberhard, Filip, \& Carlson, 2002). Similarly, verbs that disambiguate the direction of rotation in sentences about manual rotation are associated with a compatible motor response that is isolated on the verb (Zwaan \& Taylor, 2006). Additionally, an adverb that maintains focus on the action (e.g. "quickly") will show the same effect when it immediately follows the verb; however, an adverb that shifts focus to a non-action oriented element of the referential situation (e.g. "happily") does not induce motor resonance (Taylor \& Zwaan, in press). These findings offer support to the broader claim that language comprehension relies on a mental simulation of the described situation.

This is closely related to the claim that language users routinely construct representations of the situations described by language, not merely the text, and use them to derive meaning (Glenberg \& Robertson, 2000; Johnson-Laird, 1983; van Dijk \& Kintsch, 1983; Zwaan \& Radvansky, 1998). Instead of memorizing the surface features of text, language users construct representations of the events described by a text, then attempt to integrate them with one another within a given discourse. These described events remain active as a reader proceeds through text. In addition to helping a reader construct situation models for an entire discourse, they also aid in making inferences and resolving ambiguities (Zwaan \& Singer, 2003). Oftentimes, ambiguous words (such as pronouns) are retroactively disambiguated with a verb. Consider the following item ("Clinton confessed to Archie because he wanted/ offered forgiveness."), in which the verb "wanted" implies that "he" refers to Clinton while the verb "offered" implies that "he" refers to Archie (Caramazza, Grober, Garvey, \& Yates, 1977). Indeed, readers readily use information that is available after an ambiguous word in order to constrain their interpretation of the ambiguous word and to determine its meaning. Comprehension requires the integration of information within and across sentences, so if motor resonance is involved in comprehension, it should also be shown to occur as a result of meaning integration across sentences.

In the current experiment, participants read sentences containing a verb (and an immediately surrounding context) that left an element of the action ambiguous (the direction of rotation; e.g. He examined the/pie through/the microwave/window andlturned theltimer./The cookingltime neededlto bellonger [shorter].). The direction of manual rotation that the agent performs in the first sentence was not disambiguated until the final word of the second sentence. Participants were expected to use this information in order to resolve the ambiguity of the action described in the first sentence. Readers routinely construct such causal inferences during text comprehension (Graesser, Singer, \& Trabasso, 1994). Given previous data (Hauk et al., 2004; Zwaan \& Taylor, 2006), this information about the action should result in an immediate activation of the motor processes responsible for performing that action. "Longer" implies that the protagonist increased the cooking time by engaging in clockwise rotation, while "shorter" implies that the protagonist decreased the cooking time by engaging in counterclockwise rotation. The experiment is designed to test this prediction.

\section{Methods}

The method used by Zwaan and Taylor (2006; Experiment 4) was used. Participants read sentences by turning a knob continuously during the frame-by-frame presentation of a sentence. Words were presented in groups of one to three. Every $5^{\circ}$ of rotation caused a group of centrally presented words to be replaced by the next group of words in the sentence. Participants turned the knob until the last word of the sentence disappeared from the screen. On critical trials, a sentence describing an act of manual rotation (e.g. He examined thelpie through/the microwavel window andlturned theltimer./The cooking/time neededlto bellonger [or shorter]. with slashes indicating the boundaries between frames) was presented. On critical items, the first sentence included a verb that implied manual rotation, but kept the direction of rotation ambiguous. The tenth and final frame presented a word that disambiguated the direction of rotation that the protagonist performed. Participants read sentences that implied counterclockwise or clockwise manual rotation while engaging in counterclockwise or clockwise manual rotation. If our hypothesis is correct, then reading times should be faster on the critical word (the word that disambiguates the direction of the rotation mentioned in the first sentence) when the participant engages in an action that matches the direction that the critical word implies. Participants should be faster to read a word that implies clockwise rotation when they 
are turning the knob in the clockwise direction and vice versa.

\subsection{Participants}

One hundred and twenty undergraduate psychology students from Florida State University participated in the experiment for course credit. The data for three participants were eliminated due to accuracy below $70 \%$ on the comprehension questions $(M=90.6 \% \mathrm{SD}=8.8)$ and the data for two participants were eliminated because they were not native English speakers. Due to the hand-sensitivity of some of our items (see Appendix A), six left-handed participants were excluded. Five remaining participants were excluded in order to balance the number of participants in each of the eight lists. The final analysis included data from 104 participants.

\subsection{Apparatus and design}

The apparatus, design, and sentences from a previous study (Zwaan \& Taylor, 2006) were adapted for this experiment. Each item contained two sentences. The first sentence described an instance of manual rotation in which the direction was left ambiguous. The final word of the second sentence disambiguated the direction of rotation (see Appendix A). Items were presented in random order. All sentences were constructed so that they consisted of 10 frames. Two versions of each item were designed such that one version implied counterclockwise rotation while the second version implied clockwise rotation. Words were presented in black text on a white background, left justified in the center of the screen. The two versions of critical items were counter-balanced between participants.

A knob that allowed rotation-contingent, participantpaced text presentation was used in both experiments (see also Zwaan \& Taylor, 2006). The knob contained springs that returned it to the centered position when released. As the knob was turned from the center position, the computer logged a key press approximately every $5^{\circ}$. Each key press logged a reading time for a given frame of text and resulted in the presentation of the next frame. Manual rotation direction was manipulated within participants. The linguistically implied rotation direction, manual rotation direction, and the two versions of each item were counter-balanced across eight lists. There were 13 participants in each list.

\subsection{Procedure}

Participants read sentences by turning the knob in either direction (clockwise or counterclockwise). For the first half of the experiment, they turned the knob in one direction to proceed through the sentences and then switched direction for the second half. After each sentence, participants released the knob so that it returned to the center position. Each participant read 48 sentences (16 experimental, 32 fil- ler) during the experiment. A yes-or-no comprehension question pertaining to the content of the immediately preceding sentence followed half of the filler items. Participants responded to these comprehension questions using a standard keyboard.

The experiment began with a participant seated in front of a computer monitor, a keyboard, and a knob wired to the keyboard. After sitting, the participant laid the keyboard across his or her lap to answer comprehension questions. The knob remained on the desk and centered in front of the monitor for the duration of the experiment. Before the experiment began, each participant completed 20 practice trials under experimenter supervision. The experimenter made sure that participants were turning the knob smoothly throughout the duration of each sentence instead of doing the task with repetitive, jerking motions. After the practice trials, every participant was judged to be able to do the task well enough to proceed. Most participants reached this criterion after four or five practice sentences.

A trial began with the knob at the center position and the first frame of text of a sentence presented on the screen. When the participant turned the knob in the correct direction for approximately $5^{\circ}$, the second frame of text was presented. When the participant turned the knob an additional $5^{\circ}$, the third frame of text was presented. This continued until the 10th frame, at which point the participant was either instructed to release the knob and wait for the next sentence or was presented with a comprehension question. Questions required a response on the keyboard.

\section{Results and discussion}

Segment reading times less than $50 \mathrm{~ms}$ and greater than $2500 \mathrm{~ms}$ were removed from the analysis as well as reading times more than three standard deviations from a participant's cell mean. In total less than $1 \%$ of the observations were removed. All analyses used mean reading times. Initial analyses of variances (ANOVAs) with list (a betweenparticipants factor) showed that interactions between this factor and match (when the direction of a participant's manual rotation matched the direction of the manual rotation described by a sentence) all had $p$-values greater than .28 , so this factor was dropped from further analyses and $t$ tests were used (Pollatsek \& Well, 1995).

The data were subjected to a 2 (sentence region) by 2 (match) ANOVA. The main finding is a significant interaction between region and match, $F(1,103)=5.51, p=.021$ $\left[\eta_{p}^{2}=.051\right]$. This is due primarily to the fact that participants were faster to read the critical word when it implied a rotation direction that was congruent with the action they were performing as they read it (see Fig. 1), both by subjects $\left[t_{1}(103)=2.67, \quad p=.0087\right]$ and by items $\left[t_{2}(31)=2.31, p=.028\right]$.

This match advantage on the critical word supports our hypothesis because it shows that motor resonance occurs 


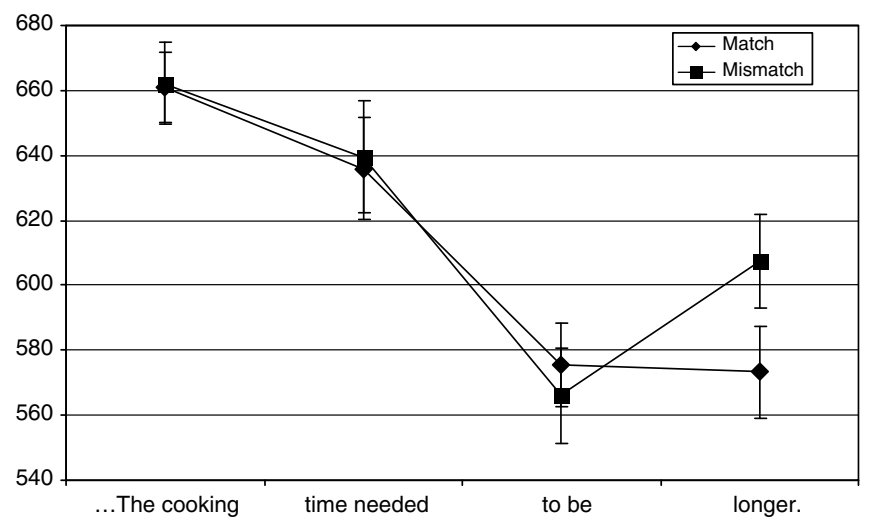

Fig. 1. Mean reading times and standard errors for the critical word and the preceding words.

on a word that, though a generated inference, disambiguates the direction of a previously mentioned action.

\section{General discussion}

The two primary issues addressed by this study concern inference-making and motor resonance during online motor processing. First, as comprehenders proceed through a text, they construct situation models of the described state of affairs. These remain active in order to be updated as the text elaborates on and resolves the ambiguities of previous events and sentences. Second, previous studies using this paradigm (e.g. Zwaan \& Taylor, 2006; Experiment 4) have focused on the relationship between the verb and compatible motor resonance during online sentence comprehension. This study shows that a word that disambiguates an element of the action, regardless of whether it is a verb, can induce motor resonance. Previous studies have supported that non-verbs can engage the action system (Gentilucci \& Gangitano, 1998; Glover et al., 2004).

The results from the experiment support the hypothesis that motor resonance is involved when a participant generates an inference about an action. When some element of an action (the direction of rotation) is left ambiguous, a word that disambiguates that element of the action will involve motor resonance for an action that is compatible with it. In this experiment, a manual rotation verb that left direction ambiguous (e.g. "turned") was presented in the first sentence. The second sentence disambiguated the direction on the critical word. Participants who read the critical word as they were engaging in a matching action responded faster than participants who read it as they were performing a mismatching action.

One alternative explanation for the results could be proposed but should be dismissed. The alternative explanation would propose that the results are due to demand effects (participants were aware of the manipulation and this drove the differences of interest). To prevent this from becoming an issue, the critical items were embedded inside a larger set of similarly worded items describing similarly mundane actions. Further, when probed during postexperiment interviews, no participant reported having any knowledge of the manipulation.

The results are in line with the claim that semantic and motor systems rely on partially overlapping neural substrates (Prinz, 1997). This broader claim converges with a claim in cognitive science suggesting that verbs denoting motor actions are partially processed in the MNS. Indeed, motor verbs activate brain regions that are associated with action (Rüschemeyer, Brass, \& Friederici, 2007) and these areas are unique from the areas activated by more "abstract," non-motor verbs (however, see Glenberg \& Kaschak, 2002 for behavioral results suggesting that the understanding of abstract transfer relies on the motor system). In line with this view, a correspondence between degeneration of the motor system and the ability to conceptually process action verbs has been noted in clinical populations (Bak et al., 2006). Similarly, when intact participants identify objects, the location of brain activity is contingent upon the intrinsic properties of the stimulus (Martin, Wiggs, Ungerleider, \& Haxby, 1996). More generally, conceptual knowledge is believed to be at least partially constrained by the modality-specific, perceptual and motor systems associated with concepts and the referents of words (Barsalou, 1999; Caramazza \& Mahon, 2006; Gallese \& Lakoff, 2005; Glenberg, 1997, 1999; Martin, 2007; for recent empirical support, see van Dantzig, Pecher, Zeelenberg, \& Barsalou, in press).

The present experiment makes two contributions to the literature on language processing. First, as a person comprehends text, she constructs a situation model of the described state of affairs. In so doing, inferences are often routinely drawn within and between sentences in order to construct a coherent representation of the described state of affairs. Making such an inference about an action involves motor resonance that is compatible with the described action. Second, previous studies demonstrating the association between comprehending text about actions and action systems have primarily focused on verbs (Taylor \& Zwaan, in press; Tettamanti et al., 2005; Zwaan \& Taylor, 2006); the present study demonstrates that motor resonance can be associated with any word that disambiguates some element of an action that a protagonist performs in text.

\section{Appendix A. The critical sentences for the experiment are listed below}

The carpenter/turned/the/screw./The boards/had/been/ connected/too/tightly (loosely).

After testing the/temperature/of the/bath water,/he turned the/cold water/faucet./The water/had been too/ hot (cold).

The technician/examined the height/of the fluid/in the test tube/he was pouring/into and adjusted/the angle./ The fluid was/flowing too/slowly (quickly). 
He put/the reading/glasses he/was holding/in his hand/ back/in place./He put them/on/his face (the table).

$\mathrm{He}$ examined the/pie through/the microwave/window and/turned the/timer./The cooking/time needed/to be/ shorter (longer).

The passenger/in the front/seat put his/hand out the/ window and/adjusted/the side mirror./It was/a little too/ high (low).

He was searching/for his favorite/radio station/by adjusting the/frequency by/turning the dial./The frequency/was a/little too/high (low).

The gardener/walked/up to/the house/and turned/the faucet./The/grass/was too/dry (wet).

When a/new song/began to play,/he approached/the stereo and/adjusted the/volume./The music/was too/loud (quiet).

The mechanic/entered/the car/and turned/the/key./ Haltingly,/the/engine/stopped (started).

As the mechanic/was replacing/the car's/tire, he/used his/hand to turn/the lug nut./It was/too/tight (loose).

The corkscrew was/halfway inserted/into the cork,/so he continued/turning it./After some/struggle, the/corkscrew was/completely/removed (inserted).

The cook/decided to/adjust the/temperature/of the oven,/so he turned/the dial./The oven/had been too/hot (cold).

After checking/the time/on her computer/she adjusted/ the time/on her/watch./It was/five minutes/fast (slow).

He was/bothered by/the amount of/light in/the room,/ so he turned/the dimmer./The room/had been too/bright (dark).

While at/the gas/station,/the driver/turned/the gas cap./ The/tank/was/empty (full).

\section{References}

Aziz-Zadeh, L., Wison, S. M., Rozzolatti, G., \& Iacoboni, M. (2006). Congruent embodied representations for visually presented actions and linguistic phrases describing actions. Current Biology, 16, 1818-1823.

Bak, T. H., Yancopoulou, D., Nestor, P. J., Xuereb, J. H., Spillantini, M. G., Pulvermüller, F., et al. (2006). Clinical, imaging and pathological correlates of a hereditary deficit in verb and action processing. Brain, 129, 321-332.

Barsalou, L. W. (1999). Perceptual symbol systems. Behavioral and Brain Sciences, 22, 577-609.

Buccino, G., Lui, F., Canessa, N., Patteri, I., Lagravinese, G., Benuzzi, F., et al. (2004). Neural circuits involved in the recognition of actions performed by nonconspecifics: An fMRI study. Journal of Cognitive Neuroscience, 16, 114-126.

Calvo-Merino, B., Glaser, D. E., Grezes, J., Passingham, P. E., \& Haggard, P. (2005). Action observation and acquired motor skills: An fMRI study with expert dancers. Cerebral Cortex, 15, 1243-1249.

Caramazza, A., \& Mahon, B. Z. (2006). The organisation of conceptual knowledge in the brain: The future's past and some future directions. Cognitive Neuropsychology, 23(1), 13-38.

Caramazza, A., Grober, E., Garvey, C., \& Yates, J. (1977). Comprehension of anaphoric pronouns. Journal of Verbal Learning and Verbal Behavior, 16, 601-609.
Chambers, C. G., Tanenhaus, M. K., Eberhard, K. M., Filip, H., \& Carlson, G. N. (2002). Circumscribing referential domains in real-time sentence comprehension. Journal of Memory and Language, 47, 30-49.

de Vega, M., Robertson, D. A., Glenberg, A. M., Kaschak, M. P., \& Rinck, M. (2004). On doing two things at once: Temporal constraints on actions in language comprehension. Memory \& Cognition, 32, 1033-1043.

di Pellegrino, G., Fadiga, L., Fogassi, L., Gallese, V., \& Rizzolatti, G. (1992). Understanding motor events: A neurophysiology study. Experimental Brain Research, 91, 176-180.

Dijkstra, K., Kaschak, M. P., \& Zwaan, R. A. (2007). Body posture facilitates retrieval of autobiographical memories. Cognition, 102, 139-149.

Fischer, M. H., \& Zwaan, R. A. (in press). Embodied language: A review of the role of the motor system in language comprehension. Quarterly Journal of Experimental Psychology.

Gallese, V. (2003). A neuroscientific grasps of concepts: From control to representation. Philosophical Transactions of the Royal Society, B, 358, $1231-1240$.

Gallese, V., Fadiga, L., Fogassi, L., \& Rizzolatti, G. (1996). Action recognition in the premotor cortex. Brain, 119, 593-596.

Gallese, V., \& Lakoff, G. (2005). The brain's concepts: The role of the sensory-motor system in reason and language. Cognitive Neuropsychology, 22, 455-479.

Gentilucci, M., \& Gangitano, M. (1998). Influence of automatic word reading on motor control. European Journal of Neuroscience, 10, $752-756$.

Gibson, J. J. (1979). The ecological approach to visual perception. Boston, MA: Houghton-Mifflin.

Glenberg, A. M. (1997). What memory is for. Behavioral and Brain Sciences, 20, 1-19.

Glenberg, A. M. (1999). Why mental models must be embodied. In G. Rickhar \& C. Habel (Eds.), Mental models in discourse processing and reasoning (pp. 77-90). Amsterdam: Elsevier Science.

Glenberg, A. M., \& Kaschak, M. P. (2002). Grounding language in action. Psychonomic Bulletin \& Review, 9, 558-565.

Glenberg, A. M., \& Robertson, D. A. (2000). Symbol grounding and meaning: A comparison of high-dimensional and embodied theories of meaning. Journal of Memory and Language, 43, 379-401.

Glover, S., \& Dixon, P. (2002). Semantics affect the planning but not control of grasping. Experimental Brain Research, 146, 383-387.

Glover, S., Rosenbaum, D. A., Graham, J., \& Dixon, P. (2004). Grasping the meaning of words. Experimental Brain Research, 154, 103-108.

Graesser, A. C., Singer, M., \& Trabasso, T. (1994). Constructing inferences during narrative text comprehension. Psychological Review, 101(3), 371-395.

Hauk, O., Johnsrude, I., \& Pulvermüller, F. (2004). Somatotopic representation of action words in human motor and premotor cortex. Neuron, 41, 301-307.

Johnson-Laird, P. N. (1983). Mental models: Towards a cognitive science of language, inference, and consciousness. Cambridge, MA: Harvard University Press.

Keysers, C., \& Perrett, D. I. (2004). Demystifying social cognition: A Hebbian perspective. Trends in Cognitive Sciences, 8, 501-507.

Martin, A. (2007). The representation of object concepts in the brain. Annual Review of Psychology, 58, 25-45.

Martin, A., Wiggs, C. L., Ungerleider, L. G., \& Haxby, J. V. (1996). Neural correlates of category-specific knowledge. Nature, 379, 649-652.

Prinz, W. (1997). Perception and action planning. European Journal of Cognitive Psychology, 9, 129-154.

Pollatsek, A., \& Well, A. D. (1995). On the use of counterbalanced designs in cognitive research: A suggestion for a better and more powerful analysis. Journal of Experimental Psychology: Learning, Memory, \& Cognition, 21, 785-794.

Pulvermüller, F., Hauk, O., Nikulin, V. V., \& Ilmoniemi, R. J. (2005). Functional links between motor and language systems. European Journal of Neuroscience, 21, 793-797. 
Rizzolatti, G., \& Arbib, M. A. (1998). Language within our grasp. Trends in Neurosciences, 21, 188-194.

Rizzolatti, G., \& Craighero, L. (2004). The mirror-neuron system. Annual Review of Neuroscience, 27, 169-172.

Rizzolatti, G., Fogassi, L., \& Gallese, V. (2001). Neurophysiological mechanisms underlying the understanding and imitation of action. Nature Reviews of Neuroscience, 2, 661-670.

Rüschemeyer, S. A., Brass, M., \& Friederici, A. D. (2007). Comprehending prehending: Neural correlates of processing verbs with motor stems. Journal of Cognitive Neuroscience, 19(5), $855-865$.

Taylor, L. J., \& Zwaan, R. A. (in press). Motor resonance and linguistic focus. Quarterly Journal of Experimental Psychology.

Tettamanti, M., Buccino, G., Saccuman, M. C., Gallese, V., Danna, M., Scifo, P., et al. (2005). Listening to action-related sentences activates frontoparietal motor circuits. Journal of Cognitive Neuroscience, 17, $273-281$.
Umiltà, M. A., Kohler, E., Gallese, V., Fogassi, L., Fadiga, L., Keysers, C., et al. (2001). "I know what you are doing": A neurophysiological study. Neuron, 32, 91-101.

Van Dijk, T. A., \& Kintsch, W. 1983. Strategies of discourse comprehension (vol. xi, pp. 418). New York: Academic Press. 0127120505. Dallas SIL Library 401.41 D575s.

van Dantzig, S., Pecher, D., Zeelenberg, R., \& Barsalou, L.W. (in press). Perceptual processing affects conceptual processing. Cognitive Science.

Wilson, M., \& Knoblich, G. (2005). The case for motor involvement in perceiving conspecifics. Psychological Bulletin, 131, 460-473.

Zwaan, R. A., \& Radvansky, G. A. (1998). Situation models in language comprehension and memory. Psychological Bulletin, 123(2), 162-185.

Zwaan, R. A., \& Singer, M. (2003). Text comprehension. In A. C. Graesser et al. (Eds.), Handbook of discourse processes (pp. 83-121). Mahwah, NJ: Lawrence Erlbaum.

Zwaan, R. A., \& Taylor, L. (2006). Seeing, acting, understanding: Motor resonance in language comprehension. Journal of Experimental Psychology: General, 135, 1-11. 